\title{
The acute myocardial infarction patient with an initially non-diagnostic electrocardiogram
}

\author{
William J Brady, Francis Morris
}

Patients presenting to the accident and emergency department with chest pain of potentially ischaemic origin are evaluated with three principle tools: the history of the event, the 12 lead electrocardiogram (ECG), and, in suspected cases of acute myocardial infarction (AMI), serum markers of cardiac injury. An accurate clinical history is pivotal in the evaluation of such patients and is a prerequisite for the correct interpretation of the ECG. The ECG is an important adjunct in the evaluation of patients with chest pain and assists the doctor in the selection of the correct management for the individual-in particular the application of treatment aimed at coronary reperfusion. Although serial ECGs are sensitive for the diagnosis of AMI, a single ECG is frequently much less helpful. In those patients who present with a clinical history compatible with AMI, fewer than $50 \%$ of patients will have the diagnostic changes of AMI on the initial ECG. The remainder will have either obvious ischaemic changes, non-specific ST segment/T wave abnormalities, or an entirely normal ECG. ${ }^{1}$ The uninformed clinician may be tempted to inappropriately discharge patients with acute chest pain if the ECG is normal or reveals only minor non-diagnostic changes.

\section{Case reports}

CASE 1

A 57 year old man presented to the accident

Department of

Emergency Medicine,

University of Virginia

School of Medicine,

Charlottesville,

Virginia, USA

W J Brady

Department of

Accident and

Emergency Medicine,

Northern General

Hospital, Sheffield

F Morris

Correspondence to:

Dr William J Brady,

Department of Emergency

Medicine, Box 523-21,

University of Virginia Health

Sciences Center,

Charlottesville, VA 22908 ,

USA (e-mail:

wb4z@hscmail.mcc.

virgina.edu).

Accepted 29 April 1999
He described a burning discomfort confined to the retrosternal area, exacerbated by exertion. The pain had started at rest and lasted 20 minutes before resolving spontaneously, before his arrival in the department. He had no prior history of ischaemic heart disease, though he was taking antihypertensive medication. Examination revealed an asymptomatic middle aged patient with no abnormal findings. A 12 lead ECG (fig 1) was normal. The patient was discharged with the diagnosis of chest pain likely resulting from a gastrointestinal source.

Approximately 18 hours after his initial visit, the patient returned with severe chest pain, nausea, diaphoresis, and dyspnoea. Clinical examination revealed a markedly diaphoretic patient with grey colour and widespread crepitations at both lung bases. The ECG (fig 2) revealed an extensive anterolateral AMI with reciprocal changes in the inferior leads. The patient received thrombolytic treatment and the ST segment elevation subsequently resolved. Creatinine phosphokinase levels were raised with positive $\mathrm{MB}$ fraction confirming AMI.

CASE 2

A previously healthy 62 year old man presented to the accident and emergency department with chest pain. The pain had first started two weeks previously. He described the pain as intensifying with exertion; on occasion, the pain was worsened with inspiration. $\mathrm{He}$ often
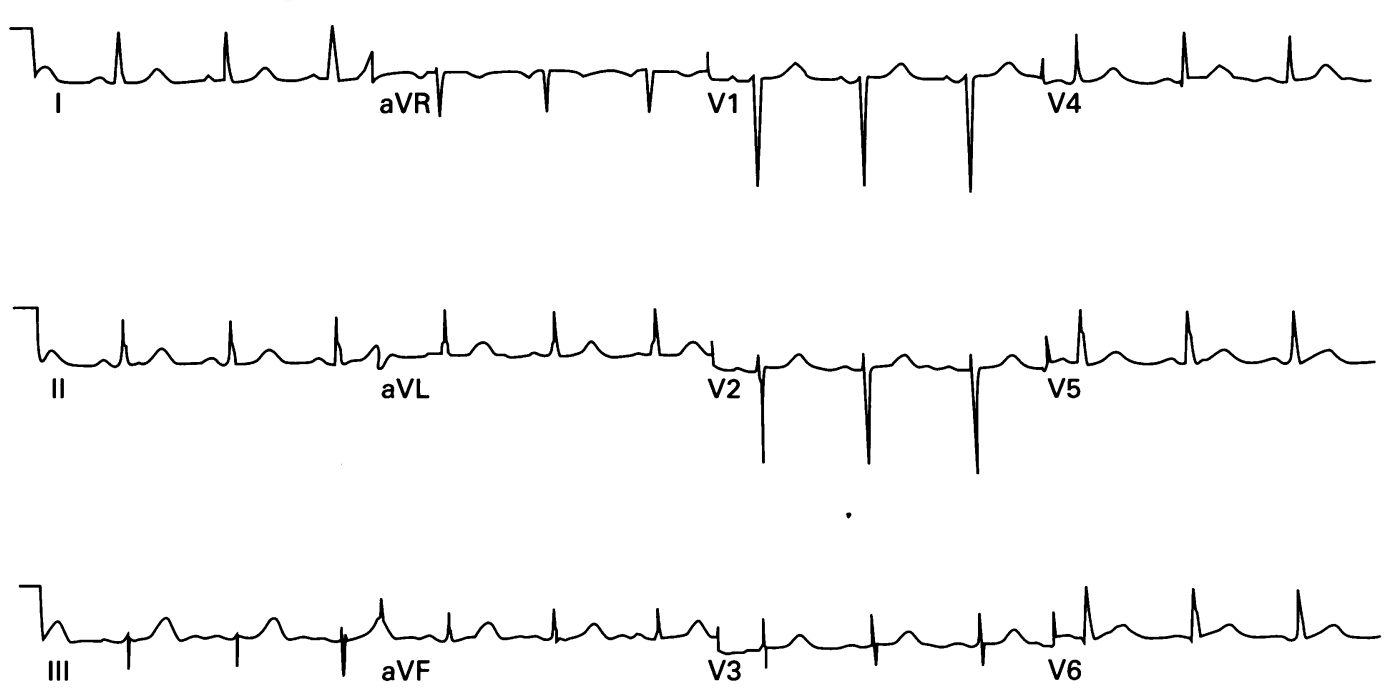

Figure 1 Normal sinus rhythm without ST segment or T wave abnormality-a normal ECG. 

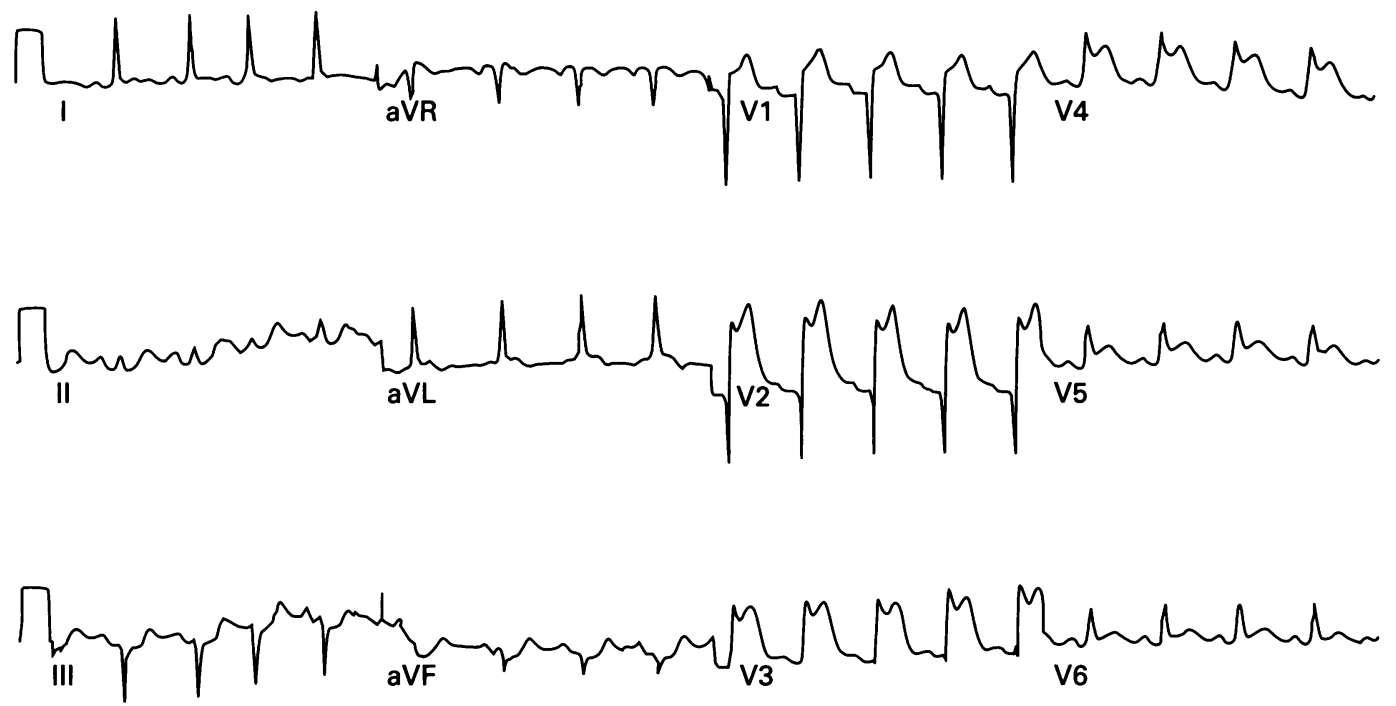

Figure 2 Normal sinus rhythm with extensive anterolateral AMI. ST segment depression-reciprocal change-is also seen in the inferior leads.

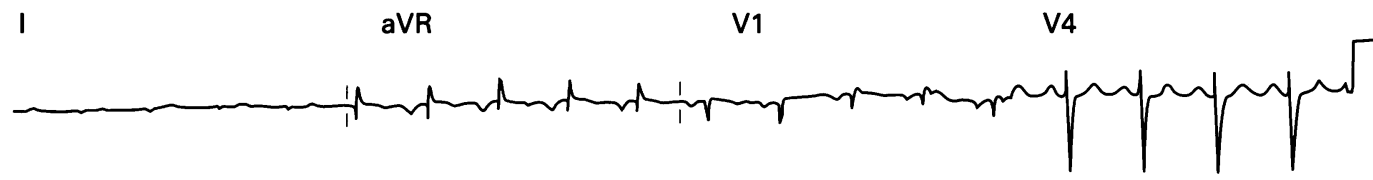

II

aVL

V2

V5

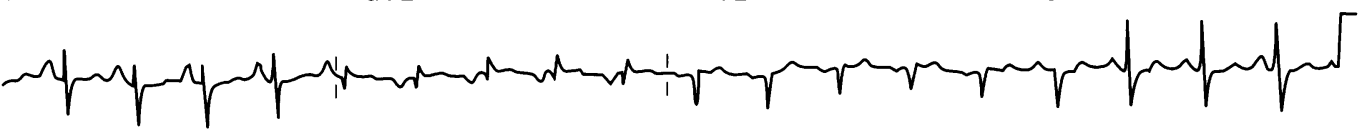

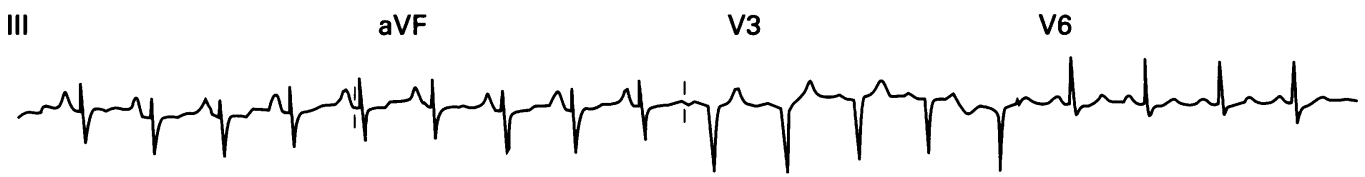

Figure 3 Normal sinus rhythm with non-specific ST segment/T wave changes. ST segment less than 1 mm is seen in the inferior and anterolateral leads while $T$ wave abnormalities are noted in the inferior and lateral leads.

felt nauseous with the pain. Clinical examination was normal except for chest wall tenderness which partially reproduced the patient's discomfort. A 12 lead ECG (fig 3) revealed normal sinus rhythm with non-specific ST segment/ $T$ wave changes evident in the inferior leads. The pain resolved spontaneously before any medical treatment, and was judged to be musculoskeletal in origin. The patient was discharged with a recommendation to use ibuprofen.
Approximately 24 hours after his initial presentation, the patient returned to the accident and emergency department with further chest pain and nausea. He appeared anxious and diaphoretic, but otherwise the examination was unremarkable. The ECG (fig 4) revealed an extensive anterolateral myocardial infarction with inferior reciprocal changes. Creatinine phosphokinase levels were raised with positive $\mathrm{MB}$ fraction confirming AMI. Coronary angiography revealed a proximal

I aVR V

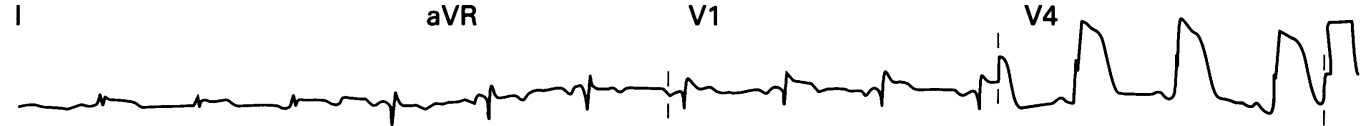

II

aVL

V2

V5

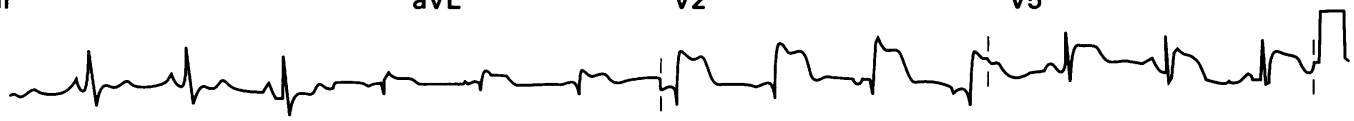

III

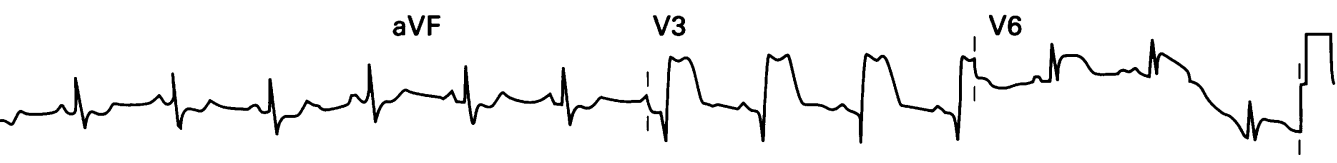

Figure 4 Normal sinus rhythm with extensive anterolateral AMI and inferior reciprocal change. 
stenosis of the left anterior descending artery which was successfully treated by balloon angioplasty. The patient subsequently had an uneventful hospital course.

\section{Discussion}

The assessment of patients with chest pain is one of the most challenging areas of accident and emergency work. Most experienced emergency physicians can recall several patients who were discharged inappropriately to complete their ischaemic event out-of-hospital. A variety of factors are frequently implicated when a patient is mismanaged. Atypical symptoms, relative inexperience on the part of the doctor, and incorrect ECG interpretation may result in patients being discharged inappropriately. To avoid this pitfall, many accident and emergency departments have adopted a strategy which centres on a very low threshold for admitting patients whose chest pain may be due to cardiac ischaemia.

The majority of patients with active coronary ischaemia will demonstrate an obviously abnormal ECG; a minority of patients, however, will have either an entirely normal or non-specifically abnormal ECG. The patient with recent resolution of the discomfort may have a normal ECG - when, in fact, the ischaemic process has resolved only temporally from both clinical and electrocardiographic perspectives. Alternatively, during active chest pain, the ECG may reveal only non-specific changes; these non-specific changes, however, may be either overlooked or felt to represent a normal ECG by the inexperienced clinician. The clinical history of the chest pain and related issues must be the primary tool used to answer disposition and therapeutic questions.

In a classic study of adult chest pain patients managed in the emergency department, Lee and colleagues found that approximately $20 \%$ of such patients had an absolutely normal 12 lead ECG. ${ }^{2}$ The description "absolutely normal" translates into the absence of non-specific $S T$ segment/ $T$ wave changes, atrioventricular block, intraventricular conduction delay, repolarisaton changes, and rhythms other sinus rhythm. The final hospital diagnoses of this group of chest pain patients with a normal 12 lead ECG in the accident and emergency department included numerous gastrointestinal, musculoskeletal, and pulmonary syndromes, as expected; this group also was composed of a minority of patients with the final hospital diagnoses of unstable angina (4\%) and AMI (1\%). A not insignificant minority of chest pain patients with an absolutely normal ECG were ultimately found to have acute coronary ischaemic syndromes. Similar issues have been reported by Rouan et al who demonstrated that patients with a normal ECG and classic symptoms of angina are at risk of an acute coronary ischaemic syndrome-with $3 \%$ of patients with final hospital diagnosis of AMI. ${ }^{3}$ Fesmire et al noted that $2 \%$ of patients ultimately found to have AMI have a normal ECG on initial presentation. ${ }^{4}$ In their report of the use of serial serum markers in the diagnosis of AMI in chest pain patients with initially non-diagnostic ECGs, Gibler et al described 69 patients ultimately diagnosed with acute infarction. ${ }^{5}$ Approximately $3 \%$ of these patients had an entirely normal ECG on initial evaluation in the accident and emergency department. In a review of patients with chest pain presenting to an accident and emergency department based chest pain centre, $3 \%$ of patients with an initially normal ECG developed AMI within 24 hours of hospital presentation (W J Brady, unpublished observation).

Additionally, it has been shown that overreliance on a normal ECG in a patient with anginal chest pain who is currently sensationfree should be avoided as well. Patients with an initially normal ECG who later develop AMI during that hospitalisation more often are sensation-free or minimally uncomfortable on presentation. It has been shown that overreliance on a normal ECG in a patient with anginal chest pain who is currently sensationfree-either due to spontaneous resolution or medical treatment-should be avoided. Further, the total elapsed time from chest pain onset in patients with normal ECGs does not assist in "ruling out" the possibility of AMI in chest pain patients with a single electrocardiographic observation. Although the negative predictive value is quite high, it is not $100 \%$ even up to 12 hours after the onset of chest discomfort. $^{6}$

The ECG may be described as "nondiagnostic" if non-specific ST segment/T wave changes are noted. These non-specific changes are defined as less than $1 \mathrm{~mm}$ ST segment depression or ST segment elevation with or without abnormal morphology and blunted, flattened, or biphasic $T$ waves without obvious inversion or hyperacuity. Other electrocardiographic issues that may produce nondiagnostic changes are sinus tachycardia and bradycardia or artifactual issues such as a wandering or irregular baseline. Lee et al noted that adult patients with chest pain with non-specific or other non-diagnostic electrocardiographic features had a relatively low risk of AMI, ranging from $3 \%$ to $4 \%$, but a significant risk of unstable angina, which occurred in approximately one fifth of all such cases. ${ }^{2}$ Fesmire et al found that $5 \%$ of patients with AMI presented with non-specific ST segment/T wave interpretations on the initial ECG. ${ }^{4}$ Gibler et al noted that $23(21 \%)$ of patients with AMI presented initially with non-specific ST segment/T wave findings on the ECG; three patients experienced $Q$ wave infarctions while the remaining 20 suffered non- $Q$ wave events. ${ }^{5}$ Other investigators have found that approximately $6 \%$ to $10 \%$ of patients with AMI demonstrate a "non-specifically abnormal" ECG on presentation (W J Brady, unpublished observation). ${ }^{3}$

Alternatively, in a somewhat different application of the term, the non-diagnostic ECG is initially encountered in $50 \%$ to $75 \%$ of patients ultimately found to have experienced a myocardial infarction. With this use of the descriptor "non-diagnostic," the clinician is referring to the lack of pathological ST segment elevation noted on the ECG. Significant ST 
segment depression and/or $\mathrm{T}$ wave changes may be seen in these situations, findings certainly suggestive of an active coronary ischaemic event.

These findings reinforce the teaching point that the history is the most important tool used in the evaluation of patients with chest pain; further, over-reliance on a normal ECG in a patient with a classic description of anginal chest pain is dangerous for the patient and the physician. Conversely, a normal ECG in a patient with a low clinical likelihood-that is, an atypical presentation-of ischaemic heart disease provides strong evidence against the possibility of acute coronary ischaemic syndrome. It must also be emphasised that the non-diagnostic-or non-specifically abnormal- ECG is exactly what the name implies: non-diagnostic. Overreliance on the non-diagnostic ECG is an approach that is fraught with both error and danger. Granted, few patients with such electrocardiographic interpretations suffer AMI, yet a sizable minority will be experiencing an unstable coronary syndrome which, if not halted, may progress on to an acute infarct or other ischaemic sequelae.

\section{Conclusions}

The default management plan for any patient presenting with acute anterior chest pain that could potentially be of ischaemic origin, who has either a normal or non-specifically abnormal ECG, should be a period of observation and re-evaluation. The justification for this type of low risk strategy comes from the understanding that those patients whose chest pain is related to acute coronary insufficiency may have either unstable angina or an evolving myocardial infarction. Recent onset ischaemic chest pain is by definition unstable and these patients are at high risk of progressing to AMI or death. In addition, alternative diagnoses such as pulmonary embolus or aortic dissection should also be considered in any patient with a normal or near normal ECG and chest pain.

The cases presented above reinforce the teaching point that the history is the most important tool in the evaluation of patients with chest pain. It must be emphasised that the ECG is a test-which is fallible. Over-reliance on either a normal or non-specifically abnormal ECG in a patient with anterior chest pain is dangerous - for both the patient and the physician. $^{7}$

Conflict of interest: none.

Funding: none.

1 Rude RE, Poole WK, Muller JE, et al. Electrocardiographic and clinical criteria for recognition of acute myocardial infarction based on analysis of 3697 patients. Am f Cardio 1983;52:936-42.

2 Lee T, Cook F, Weisberg M, et al. Acute chest pain in the emergency room: identification and examination of low risk patients. Arch Intern Med 1985;45:65-9.

3 Rouan GW, Lee TH, Cook EF, et al. Clinical characteristics of patients with acute myocardial infarction and nonspecific electrocardiograms. Clin Res 1987;35:360A.

4 Fesmire FM, Percy RF, Wears RI et al. Initial ECG in Q wave and non-Q wave myocardial infarction. Ann Emerg wed and non-Q wave

5 Gibler WB, Young GP, Hedges JR, et al. Acute myocardial infarction in chest pain patients with nondiagnostic ECGs: serial CK-MB sampling in the emergency department. $A n n$ Emerg Med 1992;21:504-12.

6 Singer AJ, Brogan GX, Valentine SM, et al. Effect of duration from symptom onset on the negative predictive value of a normal ECG for exclusion of acute myocardial infarction. Ann Emerg Med 1997;29:575-9.

7 Aufderheide TP, Brady WJ. Electrocardiography in patients with myocardial ischemia or infarction. In: Gibler WB Aufderheide TP, eds. Emergency cardiac care. St Louis: Mosby-Year Book, 1994. 